\title{
Improved Cervical Cord Lesion Detection with 3D-MP2RAGE Sequence in Patients with Multiple Sclerosis
}

\author{
(D). Demortière, (D) P. Lehmann, (D). Pelletier, (1)B. Audoin, and (D) V. Callot
} O- $\equiv$

\begin{abstract}
SUMMARY: Spinal cord lesions have a real diagnostic and prognostic role in multiple sclerosis. Thus, optimizing their detection on MR imaging has become a central issue with direct therapeutic impact. In this study, we compared the 3D-MP2RAGE sequence with the conventional Magnetic Resonance Imaging in Multiple Sclerosis (MAGNIMS) set for cervical cord lesion detection in 28 patients with multiple sclerosis. 3D-MP2RAGE allowed better detection of cervical lesions (+62\%) in this population, with better confidence, due to optimized contrast and high spatial resolution.
\end{abstract}

ABBREVIATION: MAGNIMS = Magnetic Resonance Imaging in Multiple Sclerosis

S ince the last revision of the Magnetic Resonance Imaging in Multiple Sclerosis (MAGNIMS) guidelines ${ }^{1}$ and the McDonald criteria of 2017, ${ }^{2}$ all spinal cord lesions must be counted to increase the sensitivity and specificity of the MS diagnosis. ${ }^{3}$ In this context, standardized acquisition protocols have been proposed. ${ }^{1}$ For spinal cord MR imaging, at least 2 sagittal sequences among T2WI, STIR, double inversion recovery, T1WI with gadolinium injection, and/ or T2WI axial acquisitions ${ }^{1}$ are recommended. Nonetheless, different sequences are often necessary because of motion and flux artifacts, ${ }^{4}$ which is time-consuming.

The MP2RAGE sequence ${ }^{5}$ has been shown to improve the detection of cerebral lesions that are difficult to visualize on conventional sequences, such as cortical lesions. ${ }^{6}$ This

Received October 29, 2019; accepted after revision March 23, 2020.

From the Centre d'exploration métabolique par résonance magnétique (S.D., P.L., J.P., B.A., V.C.); Departments of Neurology (S.D., J.P., B.A.); and Neuroradiology (P.L.), Assistance Publique-Hôpitaux de Marseille, Hôpital Universitaire Timone, Marseille, France; and Center for Magnetic Resonance in Biology and Medicine (V. C.), Aix-Marseille University, National Centre for Scientific Research, Marseille, France.

This work was performed in a laboratory member of the France Life Imaging network (grant ANR-11-INBS-0006) and was supported by the Fondation pour l'Aide à la Recherche sur la Sclérose en Plaques and Centre National de la Recherche Scientifique.

Paper previously presented, in part, at: Annual Meeting of the European Research Committee for Treatment and Research in Multiple Sclerosis, October 18-22; 2019; Berlin, Germany (poster p1265), for which S. Demortière held a European Research Committee for Treatment and Research in Multiple Sclerosis fellowship grant.

Please address correspondence to Virginie Callot, PhD, CRMBM-Centre d'exploration métabolique par résonance magnétique, UMR 7339, CNRS, AixMarseille Université, Faculté de Médecine, 27, bd Jean Moulin, 13385 Marseille Cedex 5, France; e-mail: virginie.callot@univ-amu.fr

- Indicates open access to non-subscribers at www.ajnr.org

Indicates article with supplemental on-line table.

http://dx.doi.org/10.3174/ajnr.A6567
T1WI MR imaging sequence, which creates a composite image, limiting field inhomogeneity bias while providing a quantitative T1 map, has recently been optimized for the spinal cord ${ }^{7}$ but has not yet been evaluated in the context of MS.

The aim of this study was to compare the MP2RAGE sequence with the conventional set of routine sequences for detecting spinal cord lesions in patients with MS.

\section{MATERIALS AND METHODS \\ Patients}

This retrospective study, approved by the local Aix-Marseille University ethics committee with written informed consent, included 28 patients with MS from January 2017 to January 2019. Inclusion criteria were a diagnosis of MS according to the revised McDonald criteria, ${ }^{2}$ older than 18 years of age, clinical symptoms suggesting spinal cord involvement, and MR imaging examination performed at least 3 months after steroid infusion.

\section{Image Acquisition}

Sequences were acquired on a 3T system (Magnetom Verio; Siemens) during the same examination. The protocol was as follows (see Table for MR imaging parameters):

- One sagittal 3D-MP2RAGE sequence, providing 2 contrasted images, from which a uniform image free of $B_{1}$ inhomogeneities and a T1 map were derived. ${ }^{5}$

- A conventional set with sagittal 2D-T2WI, STIR-weighted, and T1WI postgadolinium sequences, with axial $2 \mathrm{D}-\mathrm{T} 22^{*}$ WI MRI for confirmation of suspicious lesions on a sagittal set. 


\begin{tabular}{lllll}
\hline & \multicolumn{1}{c}{ MP2RAGE } & \multicolumn{1}{c}{ STIR-Weighted } & \multicolumn{1}{c}{ T2WI } & TIWI Postgadolinium \\
\hline Orientation/readout & 3D sagittal/GRE & 2D sagittal/TSE & 2D sagittal/TSE & 2D sagittal/TSE \\
TE/TR & $2.48 / 4000 \mathrm{~ms}$ & $53 / 4000 \mathrm{~ms}$ & $113 / 3200 \mathrm{~ms}$ & $10 / 700 \mathrm{~ms}$ \\
FOV & $300 \mathrm{~mm}$ & $320 \mathrm{~mm}$ & $280 \mathrm{~mm}$ & $220 \mathrm{~mm}$ \\
Voxel size & $0.9 \times 0.9 \times 1 \mathrm{~mm}^{3}$ & $0.9 \times 0.7 \times 3 \mathrm{~mm}^{3}$ & $1.1 \times 0.9 \times 2 \mathrm{~mm}^{3}$ & $0.8 \times 0.6 \times 3 \mathrm{~mm}^{3}$ \\
$T_{\text {acq }}$ & $7 \mathrm{~min} 18 \mathrm{sec}$ & $2 \mathrm{~min} 18 \mathrm{sec}$ & $1 \mathrm{~min} 54 \mathrm{sec}$ & $4 \mathrm{~min} 31 \mathrm{sec}$ \\
Spatial coverage & Brain $+\mathrm{Cl}-\mathrm{C} 7$ & $\mathrm{Cl}-\mathrm{C} 7$ & $\mathrm{Cl}-\mathrm{C} 7$ & $\mathrm{Cl}-\mathrm{C} 7$ \\
Phase-encoding direction & $\mathrm{A}>>\mathrm{P}$ & $\mathrm{H}>>\mathrm{F}$ & $\mathrm{H}>>\mathrm{F}$ & $\mathrm{H}>\mathrm{F}$ \\
Other parameters & TII/TI2 $=650 / 2000 \mathrm{~ms} ; \alpha \mathrm{F} / \alpha 2=4 / 5$ & & & \\
Reconstruction image & $\mathrm{T}$ quantitative map and UNI & & & \\
\hline
\end{tabular}

Note:-GRE indicates gradient recalled-echo; $\mathrm{T}_{\text {acq, }}$ acquisition time; A, anterior; P, posterior; $\mathrm{H}$, head; F, feet; UNI, uniform image; TI1/TI2, inversion times 1 and 2; $\alpha 1 / \alpha 2$, flip angles $1 / 2$.

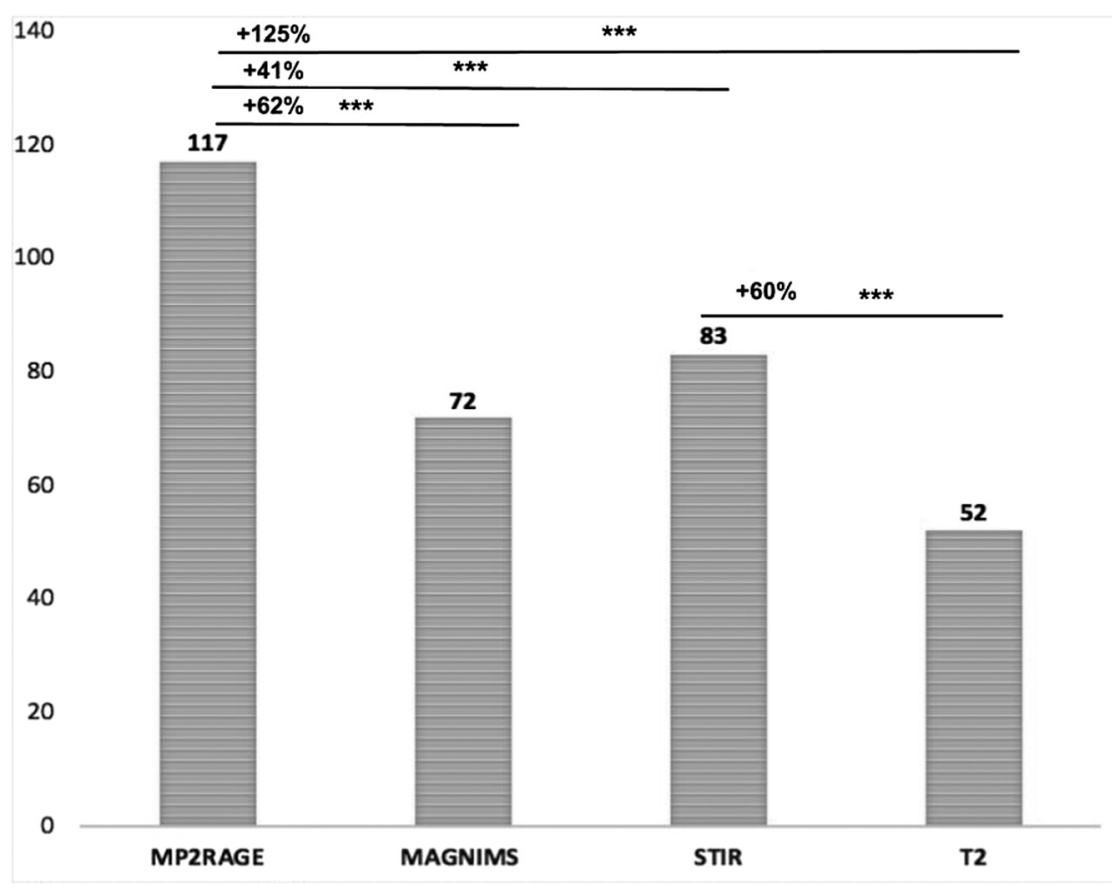

FIG 1. Number of lesions detected by sequence (Wilcoxon test; triple asterisks indicate $P<.0001$ ). The MP2RAGE sequence detected significatively more lesions than using the MAGNIMS criteria (117 vs $72, P<.0001$ ), than STIR (117 vs $83, P<.0001$ ) and T2 (117 vs $52, P<.0001$ ) sequences (according to the MAGNIMS criteria, a lesion was validated if visible on at least two sequences of the conventional set). This corresponds to a $+62 \%,+41 \%$ and $+125 \%$ increase in the lesion detection using MP2RAGE as compared to MAGNIMS, STIR and T2, respectively.

\section{Lesion Detection and Scoring Confidence}

The cervical spinal cord from C1 to C7 was evaluated. Two operators, a senior neuroradiologist and a neurologist who were blinded to patient data, read the results of the conventional set and the 3D-MP2RAGE sequence (T1 map and uniform image). Identification took place during a consensus session, ${ }^{8}$ on a highresolution monitor, using syngo.via (Siemens) image-analysis software. MS lesions were detected on the basis of hyperintensity on T2WI, STIR, MP2RAGE T1 map, and T1-weighted postgadolinium images in case of inflammatory lesions or hypointensity seen on MP2RAGE uniform images compared with normal cord signals. The lesion had to be visible on at least 2 slices in a row for the millimetric MP2RAGE sequence. According to the MAGNIMS criteria, a lesion was validated if visible on at least 2 sequences of the conventional set. Lesion detection was re- evaluated (reproducibility assessment) 3 weeks later, to minimize recall bias.

Confidence in detection was defined during the consensus session by use of a qualitative scale: ${ }^{9} 0$, no lesion detected; 1 , low detection confidence; 2 , moderate-to-high detection confidence; and 3, very high detection confidence.

\section{Statistical Analysis}

Wilcoxon tests (JMP 9; SAS Institute) were used to compare the number of detected lesions between conventional and MP2RAGE datasets, as well as the reader's confidence between sequences. $P<.05$ was considered statistically significant.

\section{RESULTS \\ Demographic and Clinical Characteristics}

We included 28 patients with MS ( 19 women; median age, 34.5 years; interquartile range, 28-41 years); 23 had relapsing-remitting MS, 4 had secondary-progressive MS, and 1 patient had primary-progressive MS. The mean Expanded Disability Status Scale score was $1.5 \pm 1.4$ (range, $0-6.5$ ). The median disease duration was 4.8 years (interquartile range, 1.7-10.1 years).

\section{Spinal Cord Lesions}

In total, 27 patients had at least 1 identifiable spinal cord lesion, and 2 patients had an active enhancing lesion. A total of 117 lesions were detected. All lesions identified during the first image-analysis session were found during the second session (100\% reproducibility). MP2RAGE significantly revealed more lesions than the conventional set $(P<.001)$ (Fig 1). All lesions seen by the conventional sagittal set were detected on MP2RAGE.

Conversely, 13 lesions (7 patients) were detected only by MP2RAGE. These lesions had an average (minimum/maximum) diameter of $2.6 \mathrm{~mm}(1.9 / 4.1 \mathrm{~mm})$. They all fulfilled the characteristics of MS lesions ${ }^{10}$ in terms of size and location (54\% posterior, 31\% 


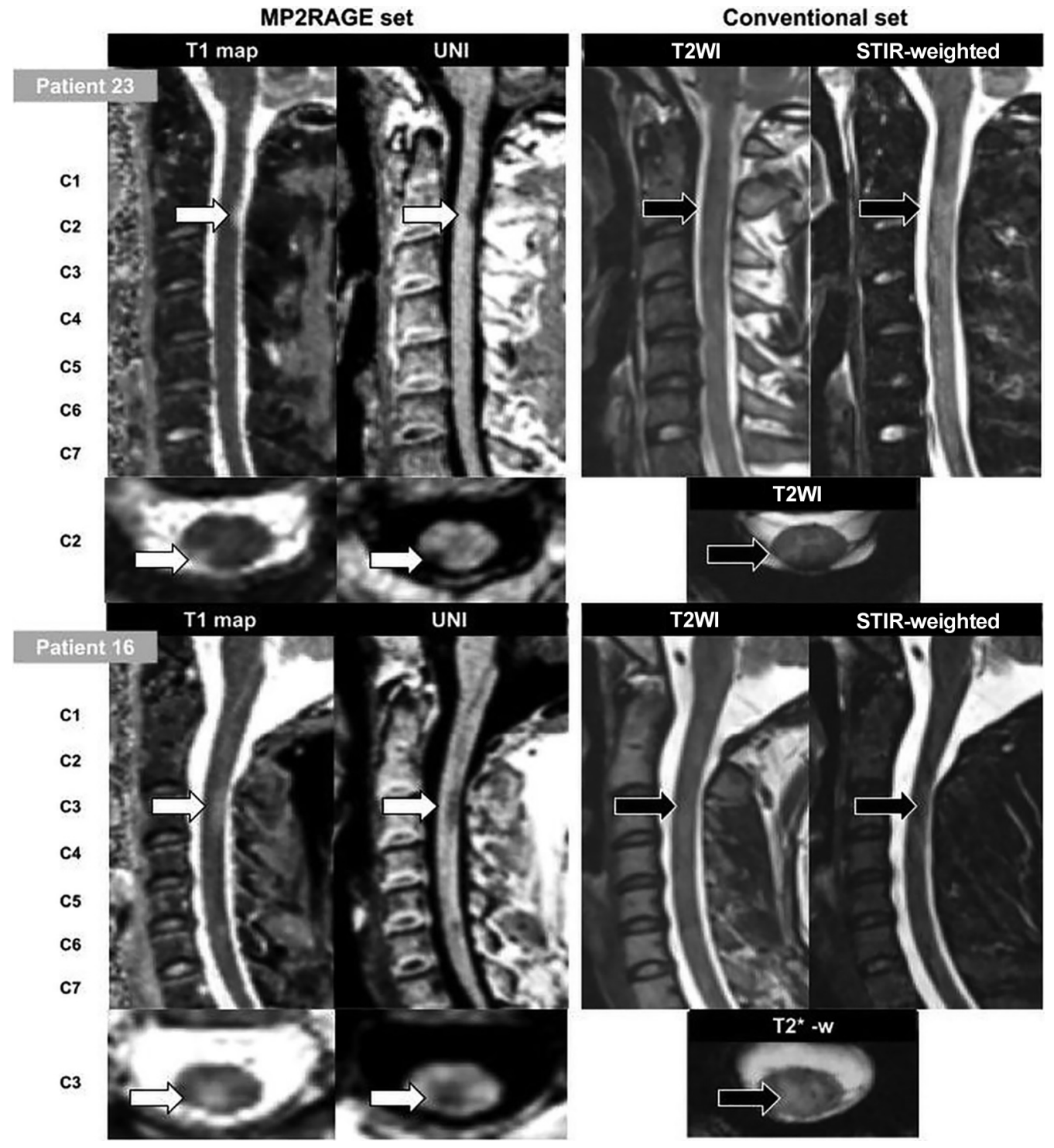

FIG 2. Illustration of MR imaging with the different investigated sequences for patient 23 (a 33-year-old man with relapsing-remitting MS) and patient 16 (a 27-year-old woman with relapsing-remitting MS). White arrows indicate good confidence in the lesion; black arrows, moderate confidence or no lesion seen; UNI, uniform image.

lateral, and 15\% anterior), and their presence was carefully doublechecked in the axial/coronal plane. The image quality of the conventional set for the corresponding slices was carefully re-evaluated and was satisfactory. Among these 7 patients, 3 had no visible lesions at all on conventional set detection (On-line Table and Fig 2).

\section{Detection Confidence}

Mean reading confidence was significantly higher with MP2RAGE than with STIR $(2.1 \pm 0.7$ versus $1.5 \pm 1.2, P=.001)$ and T2 $(2.1 \pm 0.7$ versus $0.7 \pm 0.9, P<.001)$ sequences. Confidence did not differ between lesions detected by MP2RAGE alone and those seen jointly by MP2RAGE and the conventional set $(2.0 \pm 0.6$ versus $2.2 \pm 0.8, P=.11)$.

\section{DISCUSSION}

The present study reveals, for the first time in MS, that the 3DMP2RAGE method can detect cervical cord lesions with higher confidence and higher sensitivity than the recommended conventional MAGNIMS set. Given the essential diagnostic and prognostic significance of spinal cord lesions in MS, these results could have some critical added value for clinical practice, as previously demonstrated using phase-sensitive inversion recovery or double inversion recovery contrast. ${ }^{9-11}$ In this work, 3D-MP2RAGE detected significantly more lesions than STIR, T2WI, and MAGNIMS set sequences. Most important, it also allowed detection of lesions in patients classified as not having cord lesions with the conventional set (11\%). Of note, the validation of these undetected lesions was achieved by consensus between 2 experienced operators, repeated once with blinding to the previous assessment. Furthermore, the detection confidence for all the lesions visualized by MP2RAGE alone ( $2.0 \pm 0.6, n=13$ lesions) was as high as that for those visualized by both MP2RAGE and the conventional set; this finding consolidates our findings. Finally, the characteristics of these lesions were typical for MS, ${ }^{10}$ showing, for the first time in the 
cord, similar detection capability than previously reporter in MS brain. $^{12-14}$ A recent study also demonstrated good correlation between cerebral lesions detected with MP2RAGE and histologic findings; ${ }^{6}$ however, such data were not available in the present study and similar work remains to be done.

The present study has some additional limitations. First, the sample size was small and did not include patients with clinically isolated syndrome, for whom it will be necessary to assess the potential value of MP2RAGE diagnosis. Second, the respective contrastto-noise ratios of the different sequences were not compared because of different spatial resolutions and partial volume effects. According to the present study design, we cannot exclude the possibility that the better sensitivity of MP2RAGE is mostly related to its $3 \mathrm{D}$ isotropic nature rather than its better ability to visualize lesions regardless of their size. Indeed, some authors reported a gain in sensitivity in $3 \mathrm{D}$ sequences ${ }^{15,16}$ from $28 \%$ to $65 \%$ for cervical lesion detection. ${ }^{9,11,17}$ Comparison among 3D-MP2RAGE, 3D-STIR, and $3 \mathrm{D}-\mathrm{T} 2 \mathrm{WI}$ is left for future specific studies. Concerning the 3 patients in whom no lesions were detected by the conventional set, missed lesions were mainly due to their small size and sequence spatial resolutions. However, 8/13 lesions detected only with the MP2RAGE sequence exceeded the spatial resolution of the conventional set; this feature suggests that the better sensitivity of MP2RAGE may be related more to its better contrast than its $3 \mathrm{D}$ isotropic resolution.

Finally, the MP2RAGE acquisition time (7 minutes) may seem relatively long compared with the $2 \mathrm{D}$ individual sequences. However, the volume acquired here covered the whole brain and the entire cervical spinal cord with isotropic submillimeter resolution. This feature offers promising opportunities for global assessment of the upper part of the central nervous system (brain and upper cervical cord). Brain evaluation was beyond the scope of this study. Finally, a compressed-sensing version of the sequence is now proposed, ${ }^{18}$ which may further improve the added clinical value of the MP2RAGE sequence.

Further assessment and multicentric studies must now be promoted to improve lesion-detection capabilities and clinical evaluation and bring additional proof of specificity.

\section{CONCLUSIONS}

In this study, an optimized 3D-MP2RAGE sequence was demonstrated to allow a significant gain in the detection of MS cervical lesions (including in patients otherwise presenting with no lesions on conventional MR imaging), with assessment with high reader confidence compared with the conventional set. This MP2RAGE protocol, allowing brain and cervical cord coverage in 7 minutes, could be promising to improve clinical practice. Future studies using larger samples are necessary to confirm these preliminary results.

\section{ACKNOWLEDGMENTS}

The authors thank V. Gimenez, L. Pini, C. Costes, and M.P. Ranjeva for study logistics; A. Rico for patient recruitment; A. Massire, J.P. Ranjeva, and A. Maarouf for helpful discussions; and T. Kober (Siemens) for MP2RAGE sequence provision.

Disclosures: Sarah Demortière-RELATED: Grant. France Life Imaging Network.* Jean Pelletier-UNRELATED: Board Membership: Roche, Merck, Biogen, Novartis*;
Consultancy: Roche, Merck, Biogen, Novartis*; Grants/Grants Pending: Fondation pour l'Aide à la recherche sur la Sclérose en Plaques Roche, Merck*; Payment for Lectures Including Service on Speakers Bureaus: Novartis, Biogen, Roche, Merck.* Virginie Callot-RELATED: Grant: Accueil-Fondation Sclérose en plaques, Comments: grant support for MRI exams. *Money paid to the institution.

\section{REFERENCES}

1. Filippi M, Rocca MA, Ciccarelli O, et al. MRI criteria for the diagnosis of multiple-sclerosis: MAGNIMS consensus guidelines. Lancet Neurol 2016;15:292-303 CrossRef Medline

2. Thompson AJ, Banwell BL, Barkhof F, et al. Diagnosis of multiple sclerosis: 2017 Revisions of the McDonald Criteria. Lancet Neurol 2018;17:162-73 CrossRef Medline

3. Filippi M, Preziosa P, Meani A, et al. Prediction of a multiple-sclerosis diagnosis in patients with clinically isolated syndrome using the 2016 MAGNIMS and 2010 McDonald criteria: a retrospective study. Lancet Neurol 2018;17:133-42 CrossRef Medline

4. Vargas MI, Delavelle J, Kohler R, et al. Brain and spine MRI artifacts at 3 Tesla. J Neuroradiol 2009;36:74-81 CrossRef Medline

5. Marques JP, Kober T, Krueger G, et al. MP2RAGE, a self bias-field corrected sequence for improved segmentation and T1-mapping at high field. Neuroimage 2010;49:1271-81 CrossRef Medline

6. Beck ES, Sati P, Sethi V, et al. Improved visualization of cortical lesions in multiple-sclerosis using 7T MP2RAGE. AJNR Am J Neuroradiol 2018;39:459-66 CrossRef Medline

7. Rasoanandrianina H, Massire A, Taso M, et al. Regional T1 mapping of the whole cervical spinal cord using an optimized MP2RAGE sequence. NMR.Biomed 2019;32:e4142 CrossRef Medline

8. Poonawalla AH, Hou P, Nelson FA, et al. Cervical spinal cord lesions in multiple-sclerosis: T1-weighted inversion-recovery MR imaging with phase-sensitive reconstruction. Radiology 2008;246:258-64 CrossRef Medline

9. Fechner A, Savatovsky J, El Methni J, et al. A 3T phase-sensitive inversion recovery MRI sequence improves detection of cervical spinalcord lesions and shows active lesions in patients with multiple-sclerosis. AJNR Am J Neuroradiol 2019;40:370-75 CrossRef Medline

10. Moccia M, Ruggieri S, Ianniello A, et al. Advances in spinal-cord imaging in multiple-sclerosis. Ther Adv Neurol Disord 2019;12:1756286419840593 CrossRef Medline

11. Mirafzal S, Goujon A, Deschamps R, et al. 3D PSIR MRI at 3 Tesla improves detection of spinal-cord lesions in multiple-sclerosis. $J$ Neurol 2020;267:406-14 CrossRef

12. Kober T, Granziera C, Ribes D, et al. MP2RAGE multiple-sclerosis magnetic resonance imaging at 3 T. Invest Radiol 2012;47:346-52 CrossRef Medline

13. Fartaria MJ, Bonnier G, Roche A, et al. Automated detection of white matter and cortical lesions in early stages of multiple-sclerosis. J Magn Reson Imaging 2016;43:1445-54 CrossRef Medline

14. Fartaria MJ, Sati P, Todea A, et al. Automated detection and segmentation of multiple-sclerosis lesions using ultra-high-field MP2RAGE. Invest Radiol 2019;54:356-64 CrossRef Medline

15. Nayak NB, Salah R, Huang JC, et al. A comparison of sagittal short $\mathrm{T} 1$ inversion recovery and T2-weighted FSE sequences for detection of multiple-sclerosis spinal cord lesions. Acta Neurol Scand 2014;129:198-203 CrossRef Medline

16. Chong AL, Chandra RV, Chuah KC, et al. Proton density MRI increases detection of cervical spinal-cord multiple-sclerosis lesions compared with T2-weighted fast spin-echo. AJNR Am J Neuroradiol 2016;37:180-84 CrossRef Medline

17. Riederer I, Karampinos DC, Settles M, et al. Double inversion recovery sequence of the cervical spinal-cord in multiple sclerosis and related inflammatory diseases. AJNR Am J Neuroradiol 2015;36:21925 CrossRef Medline

18. Mussard E, Hilbert T, Forman C, et al. Accelerated MP2RAGE imaging using Cartesian phyllotaxis readout and compressed sensing reconstruction. Magn Reson Med 2020 CrossRef Medline 